\title{
WISSENSCHAFT UND KULTUR
}

BAND 9 
ANDREAS SPEISER

PROFESSOR AN DER UNIVERSITÄT BASEL

\section{DIE \\ GEISTIGE ARBEIT}

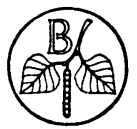

SPRINGER BASEL AG 
ISBN 978-3-0348-6939-3 ISBN 978-3-0348-6938-6 (eBook)

DOI 10.1007/978-3-0348-6938-6

Nachdruck verboten. Alle Rechte vorbehalten, insbesondere das der Übersetzung in fremde Sprachen und der Reproduktion auf photostatischem Wege oder durch Mikrofilm

(C) Springer Basel AG 1955

Ursprünglich erschienen bei Birkhäuser Verlag Basel 1955

Softcover reprint of the hardcover ist edition 1955 


\section{INHALTSVERZEICHNIS}

\section{Vorwort}

I. Die geistige Arbeit . . . . . . . . . . I

2. Die räumliche Deutung der Aussenwelt . . . I 17

3. Die Platonische Lehre vom unbekannten Gott und die christliche Trinität . . . . . . . 32

4. Platons Ideenlehre und die Mathematik . . . . 45

5. Wissenschaft und Glaube . . . . . . . . 65

6. Der Erlösungsbegriff bei Plotin . . . . . . . 82

7. Die mathematische Betrachtung der Kunst . . . 96

8. Die Gottesbeweise von Plato, Anselmus und Leibniz Io9

9. Die philosophisch-naturwissenschaftliche Fakultät • I24

10. Geist und Mathematik . . . . . . . . . I39

II. Die Grundlagen der Mathematik von Plato bis Fichte IS I

12. Über die Freiheit . . . . . . . . . . . I74

13. Die mathematische Denkweise in den Geisteswissenschaften und in der Kunst . . . . . . . . . 189

Quellennachweis . . . . . . . . . 207 


\section{VORWORT}

Die Vorträge und Abhandlungen, die in diesem Bändchen vereinigt sind, gehören inhaltlich beiden Sektionen der philosophischen Fakultät an, nämlich der philologisch-historischen einerseits und der mathematisch-naturwissenschaftlich anderseits, und es wird versucht, die Kluft, die durch die unheilvolle Spaltung entstanden ist, zu überbrücken. Wie der Körper eine gewisse Zeit mit einseitiger Nahrung auskommt, so geht es auch dem Geist. Aber mit der Zeit machen sich Schäden bemerkbar. Die Mathematik ist ein wesentlicher Bestandteil des nutrimentum spiritus und heute ist es wohl allgemein anerkannt, dass man in der Wissenschaft und in der Kunst mit der blossen Historie nicht auslangt. Anderseits hat sich die Mathematik allzusehr isoliert, und es ist nötig, ihre neueren Errungenschaften auf die Brauchbarkeit in den übrigen Geistesgebieten zu prüfen. Früher, als Kunst und Wissenschaft nicht getrennt waren, ging alles gleichmässig vonstatten.

Den etwas anspruchsvollen Titel bitte ich zu verzeihen, er stammt aus meiner Antrittsvorlesung in Basel und ich habe ihn beibehalten, weil er kurz ist und auf das übrige passt. Auch konnte ich es nicht vermeiden, dass einige Gedanken mehrfach vorkommen, so namentlich die Gotteslehre von Plato. Gerade diese reicht so hoch in die geistige Region hinauf, dass man sie von manchen Seiten notwendig sehen muss, und stets bietet sie dem Beschauer einen neuen Anblick dar. Alle Polemik habe ich streng vermieden. Sollte doch etwas derartiges zurückgeblieben sein, so bitte ich auch dafür um Verzeihung.

Dem Birkhäuser Verlag spreche ich den herzlichsten Dank aus, dass er dieses Buch aufgenommen hat und die farbige Figur auf dem Umschlag hinzufügte. Sie ist das Ergebnis einer Arbeit von über hundert Jahren und trägt den Namen «Die Kleinsche Kreisfigur», weil FELIx KLEIN sie vor achtzig Jahren entdeckte und ihre Bedeutung für die Kunst voraussah.

Basel, den 10. September 1955.

A. SPEISER 